\title{
Near-complete teleportation of a superposed coherent state
}

\author{
Yong Wook Cheong, Hyunjae Kim, and Hai-Woong Lee \\ Department of Physics, Korea Advanced Institute of Science and Technology, Daejeon 305-701, Korea
}

(Received 5 September 2003; published 28 September 2004)

\begin{abstract}
The four Bell-type entangled coherent states, $|\alpha\rangle|-\alpha\rangle \pm|-\alpha\rangle|\alpha\rangle$ and $|\alpha\rangle|\alpha\rangle \pm|-\alpha\rangle|-\alpha\rangle$, can be discriminated with a high probability using only linear optical means, as long as $|\alpha|$ is not too small. Based on this observation, we propose a simple scheme to almost completely teleport a superposed coherent state. The nonunitary transformation that is required to complete the teleportation can be achieved by embedding the receiver's field state in a larger Hilbert space consisting of the field and a single atom and performing a unitary transformation on this Hilbert space.
\end{abstract}

DOI: 10.1103/PhysRevA.70.032327

PACS number(s): 03.67.Hk, 03.65.Ud, 42.50.-p

\section{INTRODUCTION}

Since its first proposal [1], a large number of theoretical and experimental investigations have shown that teleportation of superposed polarization states [2], superposed oneparticle and vacuum states [3], and continuous-variable light states [4] can be accomplished. The major obstacle to a demonstration of a complete teleportation for discrete systems with $100 \%$ success probability is that a clear distinction of four Bell states, a necessary requirement for a successguaranteed teleportation, is not possible with linear optical means [5]. Although this obstacle can in principle be overcome by various means such as exploiting feedback from detectors, employing additional degrees of freedom or utilizing nonlinear optical interactions [6], its experimental realization seems difficult [7].

Quantum teleportation of a superposed coherent state (known as the Schrödinger cat state), a superposition of two nonorthogonal coherent states with opposite phases, has also been studied in the past [8]. Theoretical investigations have revealed that an interesting feature that characterizes teleportation of a superposed coherent state is that the four "quasiBell states," four Bell-type entangled coherent states [9], can be distinguished with a probability approaching unity using only linear optical devices. The main purpose of this work is to propose a simple scheme that can perform a near-complete teleportation of a superposed coherent state with the success probability and fidelity of nearly $100 \%$. The scheme uses linear optics except in the state transformation process that needs to be carried out in the final stage of the teleportation, where an additional system, an atom, is brought in contact with the field and the subsequent atom-field interaction is utilized.
It should be emphasized that, while the teleportation scheme for continuous-variable light states (in which a $100 \%$ success probability can in principle be achieved through ideal homodyne detection [4]) is designed to teleport the quadrature amplitudes of a light field, our scheme takes a discrete system with the coherent states of opposite phases, $|\alpha\rangle$ and $|-\alpha\rangle$, as the two basis states for a qubit. An input state for the continuous-variable teleportation is typically a coherent state, whereas for our teleportation scheme it is a superposed coherent state.

\section{TELEPORTATION SCHEME}

The scheme we propose is identical to the standard linearoptical teleportation scheme, except that Bob's station needs to be equipped with an additional system, i.e., a single atom trapped in a cavity, to perform a state transformation, as described later. At the source station, the entangled coherent state

$$
|\Psi\rangle_{A B}=N\left(|\alpha\rangle_{A}|-\alpha\rangle_{B}-|-\alpha\rangle_{A}|\alpha\rangle_{B}\right)
$$

is generated, where $A$ and $B$ refer to the waves that are sent to Alice and Bob, respectively, and $N\left[=1 / \sqrt{2\left(1-e^{-4|\alpha|^{2}}\right)}\right]$ is the normalization constant. At Alice's station, the wave $A$ is combined via a 50/50 beam splitter with another wave $C$ that contains an unknown superposed coherent state to be teleported,

$$
|\Psi\rangle_{C}=x|\alpha\rangle_{C}+y|-\alpha\rangle_{C},
$$

where the unknown coefficients $x$ and $y$ satisfy the normalization condition ${ }_{C}\langle\Psi \mid \Psi\rangle_{C}=1$. The state $|\Psi\rangle_{A B}|\Psi\rangle_{C}$ is transformed, by the action of the beam splitter, into the state $|\Psi\rangle_{E F B}$,

$$
\begin{aligned}
|\Psi\rangle_{E F B}= & \frac{N}{2}\left\{-|0\rangle_{E}\left(|\sqrt{2 \alpha}\rangle_{F}-|\sqrt{-2 \alpha}\rangle_{F}\right)\left(x|\alpha\rangle_{B}+y|-\alpha\rangle_{B}\right)-|0\rangle_{E}\left(|\sqrt{2 \alpha}\rangle_{F}+|\sqrt{-2 \alpha}\rangle_{F}\right)\left(x|\alpha\rangle_{B}-y|-\alpha\rangle_{B}\right)+\left(|\sqrt{2 \alpha}\rangle_{E}-|\sqrt{-2 \alpha}\rangle_{E}\right)|0\rangle_{F}(x \mid\right. \\
& \left.\left.-\alpha\rangle_{B}+y|\alpha\rangle_{B}\right)+\left(|\sqrt{2 \alpha}\rangle_{E}+|\sqrt{-2 \alpha}\rangle_{E}\right)|0\rangle_{F}\left(x|-\alpha\rangle_{B}-y|\alpha\rangle_{B}\right)\right\}
\end{aligned}
$$


where $E$ and $F$ denote the waves that exit through the two output ports of the beam splitter, which lead to the detectors $D_{E}$ and $D_{F}$, respectively. Equation (3) indicates that, as has already been noted [8], the four "quasi-Bell states," $(|\alpha\rangle \mid$ $-\alpha\rangle \pm|-\alpha\rangle|\alpha\rangle)$ and $(|\alpha\rangle|\alpha\rangle \pm|-\alpha\rangle|-\alpha\rangle)$, can be distinguished by observing which detector, $D_{E}$ or $D_{F}$, measures an odd or even number of photons and which detects no photon. The only indistinguishable case is when both detectors detect no photon, in which case no distinction can be made between $(|\alpha\rangle|-\alpha\rangle+|-\alpha\rangle|\alpha\rangle)$ and $(|\alpha\rangle|\alpha\rangle+|-\alpha\rangle|-\alpha\rangle)$. The probability that Alice's Bell measurement fails is then given by $P_{F}$ $=\left.\right|_{F} \mid\left\langle\left.\left. 0\right|_{E}\langle 0 \mid \Psi\rangle_{E F B}\right|^{2}=\left.\left.\right|_{C}\left\langle\left. 0\right|_{A}\langle 0 \mid \Psi\rangle_{A B} \mid \Psi\right\rangle_{C}\right|^{2}\right.$ and can easily be calculated to be

$$
P_{F}=P_{F}(x, y)=\frac{e^{-2|\alpha|^{2}}}{1+e^{-2|\alpha|^{2}}}|x+y|^{2} .
$$

This is negligible for sufficiently large $|\alpha|$.

After being informed of Alice's Bell measurement result, Bob needs to perform an appropriate transformation on the field state $B$ to complete the teleportation. If detector $D_{E}$ detects no photon and detector $D_{F}$ measures an odd number of photons, corresponding to the first term of Eq. (3), then Bob need do nothing and the teleportation is achieved. If detector $D_{E}$ measures an odd number of photons and detector $D_{F}$ detects no photon, corresponding to the third term of Eq. (3), then the unitary transformation $(-1)^{a^{\dagger} a}$ brings the state of the field $B$ to the original state.

The difficulty arises when either of the two detectors measures a nonzero even number of photons, because then a nonunitary transformation is needed. This nonunitary transformation can be accomplished approximately by applying a unitary transformation that displaces the state by an appropriate amount [10], or probabilistically by performing teleportation repeatedly with entangled ancilla photons until the desired transformation is reached [11]. We show in the next section that one can perform the required nonunitary transformation successfully with a high probability by bringing an additional system, a single atom trapped in a cavity, in contact with the field to be transformed and utilizing the interaction between the field and the atom.

\section{STATE TRANSFORMATION}

Let us consider the case when detector $D_{E}$ detects no photon and $D_{F}$ measures a nonzero even number of photons. The transformation needed is $M(x|\alpha\rangle-y|-\alpha\rangle) \Rightarrow(x|\alpha\rangle+y|-\alpha\rangle)$, where $M$ is the normalization constant $[M$ $\left.=1 / \sqrt{2\left(|x|^{2}+|y|^{2}\right)-1}\right]$. In order to achieve this transformation, we allow the field to interact with a single two-level atom prepared in its lower level $|g\rangle$. We assume that the atomic transition between the upper level $|e\rangle$ and the lower level $|g\rangle$ is resonant with the field frequency. The state of the system, atom +field, at the initial time, $t=0$, is $|\psi(t=0)\rangle$ $=M|g\rangle(x|\alpha\rangle-y|-\alpha\rangle)$. The state at a later time $t>0$ is given, under the rotating-wave approximation, by the solution of the Jaynes-Cummings model, provided that spontaneous emission and the cavity decay can be neglected. We then obtain, for the fidelity of the field state at time $t$ with respect to the desired state $(x|\alpha\rangle+y|-\alpha\rangle)$,

$$
\begin{aligned}
F(x, y)= & \left(x ^ { * } \left\langle\alpha\left|+y^{*}\langle-\alpha|\right) \rho(x|\alpha\rangle+y|-\alpha\rangle)=|M|^{2} e^{-2|\alpha|^{2}}\left\{\left|\sum_{n=0}^{\infty} \frac{|\alpha|^{2 n}}{n !}\left[|x|^{2}-|y|^{2}+2(-1)^{n} i \operatorname{Im}\left(x y^{*}\right)\right] \cos \left(\frac{\sqrt{n} g_{0} t}{2}\right)\right|^{2}\right.\right.\right. \\
& \left.+\left|\sum_{n=0}^{\infty} \frac{|\alpha|^{2 n+1}}{\sqrt{n !(n+1) !}}\right| x+\left.\left.(-1)^{n} y\right|^{2} \sin \left(\frac{\sqrt{n+1} g_{0} t}{2}\right)\right|^{2}\right\},
\end{aligned}
$$

where $\rho$ is the reduced density operator of the field at time $t$, and $g_{0}$ is the single-photon Rabi frequency. The fidelity $F(x, y)$ depends upon $x$ and $y$ as well as $\alpha$ and $t$. In Figs. 1(a) and $1(\mathrm{~b})$, we show the fidelity $F$ we computed as a function of time for the case $\alpha=5$ and for $x=y=1 / \sqrt{2\left(1+e^{-50}\right)}$ $\simeq 1 / \sqrt{2}$, and $x=\sqrt{2} y=\sqrt{2 /\left(3+2 \sqrt{2} e^{-50}\right)} \simeq \sqrt{2 / 3}$, along with the probability $P_{e}$ at time $t$ that the atom is found in its upper level $|e\rangle$. It is seen that $F$ and $P_{e}$ show similar temporal behavior. The time at which $F$ takes on the largest value is given roughly by $t \simeq \pi /|\alpha| g_{0}$, as long as $|\alpha|$ is not too small $(|\alpha| \geq 3)$. Figure 2 shows $F\left(t=\pi /|\alpha| g_{0}\right)$, the fidelity $F$ at $t$ $=\pi /|\alpha| g_{0}$, as a function of $\alpha$ for the case $x=y$ $=1 / \sqrt{2\left(1+e^{-2|\alpha|^{2}}\right)}$ (when $|\Psi\rangle_{c}$ is the even coherent state) and for the case $x=-y=1 / \sqrt{2\left(1-e^{-2|\alpha|^{2}}\right)}$ (when $|\Psi\rangle_{c}$ is the odd coherent state). It is seen that, as $|\alpha|$ becomes larger, the fidelity at $t=\pi /|\alpha| g_{0}$ becomes close to unity. We note that the fidelity $F\left(t=\pi /|\alpha| g_{0}\right)$ varies slowly with respect to $x$ and $y$ for $|\alpha| \gtrsim 1$. For example, the plot for $F\left(t=\pi /|\alpha| g_{0}\right)$ for the case $x=\sqrt{2} y=\sqrt{2 /\left(3+2 \sqrt{2} e^{-50}\right)}$ and for the case $x=-\sqrt{2} y$ $=\sqrt{2 /\left(3-2 \sqrt{2} e^{-50}\right)}$ is almost indistinguishable from Fig. 2. It should be remarked that, when we fix the values of $\alpha, x$, and $y$ and follow the time variation of the fidelity $F$, the maximum value $F_{\max }$ occurs at the time very close to but not exactly equal to $\pi /|\alpha| g_{0}$. Thus, the actual maximum value $F_{\max }$ of the fidelity is slightly greater than the value $F(t$ $\left.=\pi /|\alpha| g_{0}\right)$ that Fig. 2 indicates. The difference, however, is negligibly small if $|\alpha| \geqslant 3$. One can conclude that, as long as $|\alpha|$ is not too small $(|\alpha| \geqslant 3)$, the transformation of the field 
(a)

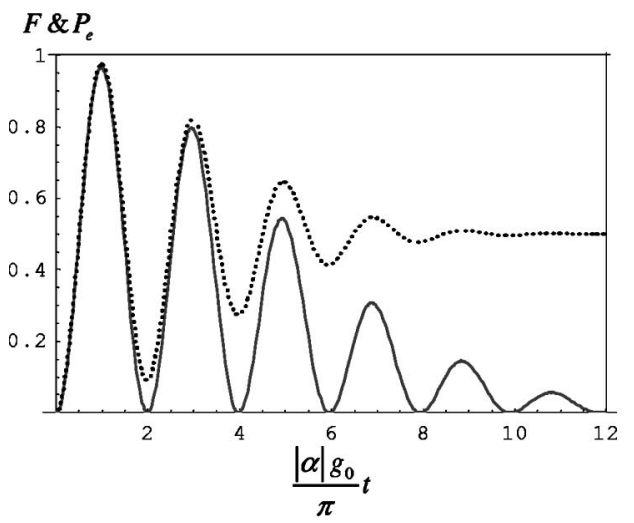

(b)

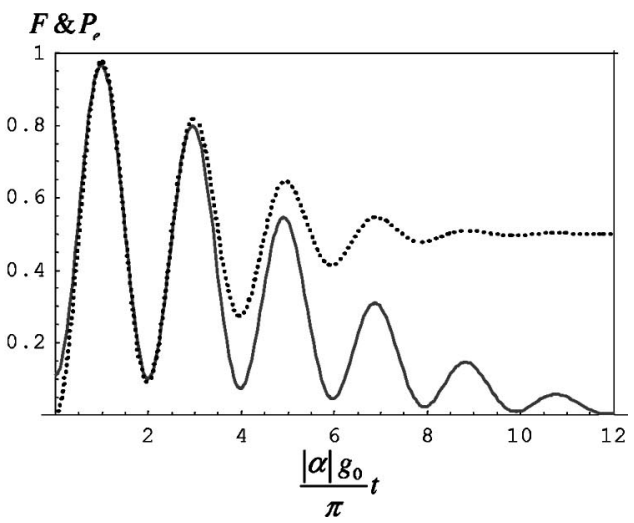

FIG. 1. The fidelity $F$ (solid curve) of Eq. (5) and the excitation probability $P_{e}$ (dotted curve) as a function of time for the case $\alpha=5$ and (a) $x=y=1 / \sqrt{2\left(1+e^{-50}\right)}$, (b) $x=\sqrt{2} y=\sqrt{2 /\left(3+2 \sqrt{2} e^{-50}\right)}$. state from $M(x|\alpha\rangle-y|-\alpha\rangle)$ to $(x|\alpha\rangle+y|-\alpha\rangle)$ can be achieved with high fidelity by allowing the field to interact with a single two-level atom in its lower level and waiting for a time of $\pi /|\alpha| g_{0}$ (i.e., waiting until the atom is excited). At this instant, if one wishes, one can measure the state of the atom, decoupling the atom from the field, and confirm that the atom has indeed been excited.

When detector $D_{E}$ measures a nonzero even number of photons and detector $D_{F}$ detects no photon, Bob needs to first apply the unitary transformation $(-1)^{a^{\dagger} a}$ to the field state and then allow the field to interact with a single atom as described above.

We remark that, as our analysis of the atom-field interaction neglects spontaneous emission and the cavity decay, the successful operation of our scheme requires a setting in which coherent atom-field interaction dominates dissipation. Specifically, it requires $\sqrt{\bar{n}} g_{0} \gg \gamma$ and $\sqrt{\bar{n}} g_{0} \gg \bar{n} \kappa$, where $n^{-}$ $\left(=|\alpha|^{2}\right)$ is the average photon number in the field, $\gamma$ the spontaneous decay rate of the atom, and $\kappa$ the cavity field decay

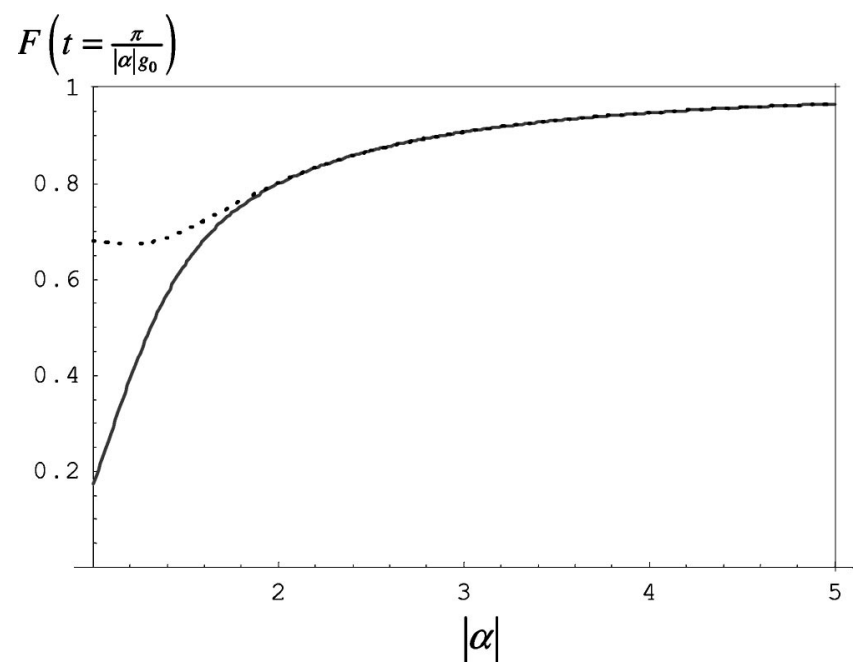

FIG. 2. The fidelity $F\left(t=\pi /|\alpha| g_{0}\right)$ as a function of $|\alpha|$ for the case $x=y=1 / \sqrt{2\left(1+e^{-2|\alpha|^{2}}\right)}$ (solid curve) and for the case $x=-y$ $=1 / \sqrt{2\left(1-e^{-2|\alpha|^{2}}\right)}$ (dotted curve). rate. We call attention to the factor $\bar{n}$ on the right-hand side of the second inequality, which arises because the superposed coherent state interacting with an atom in a cavity decoheres fast into a statistical mixture in time $\sim 1 / \bar{n} \kappa$ $[12,13]$. Because of this factor, strong coherent fields cannot be used in our scheme. As an example, let us take the experimental parameters, $g_{0} / 2 \pi=47 \mathrm{KHz}, \tau_{s p}=1 / \gamma=30 \mathrm{~ms}$, $\tau_{c}=1 / \kappa=1 \mathrm{~ms}$, quoted in a recent cavity quantum electrodynamics (QED) experiment with circular Rydberg states of rubidium atoms in a millimeter wave super-conducting highfinesse cavity [14]. The first inequality is easily satisfied, but the second inequality requires $\sqrt{\bar{n}} \ll g_{0} / \kappa \approx 300$, i.e., $\bar{n} \ll 10^{5}$. If we take $g_{0} / 2 \pi=32 \mathrm{MHz}, \gamma / 2 \pi=2.6 \mathrm{MHz}$, and $\kappa / 2 \pi$ $=4 \mathrm{MHz}$ from an experiment [15] conducted in the nearinfrared regime with cesium atoms trapped in a far-offresonance trap, the first inequality requires $\sqrt{\bar{n}} \gg \gamma / g_{0}$ $\approx 0.081$, i.e., $\bar{n} \gg 0.066$, and the second requires $\sqrt{\bar{n}} \ll g_{0} / \kappa$ $=8$, i.e., $\bar{n} \ll 64$.

\section{AVERAGE FIDELITY}

We now wish to calculate the average fidelity of the entire teleportation process. The average fidelity $F_{\text {av }}$ can be defined as

$$
F_{\mathrm{av}}=\sum_{i=1}^{5} \overline{P_{i}(x, y) F_{i}(x, y)},
$$

where $P_{1}, P_{2}, P_{3}, P_{4}$, and $P_{5}$ represent, respectively, the probability that the number of photons measured by detectors $\left(D_{E}, D_{F}\right), \quad$ is $(0$, odd $), \quad($ odd, 0$), \quad(0$, nonzero-even $)$, (nonzero-even, 0$)$, and $(0,0) ; F_{i}$ denotes the fidelity of the field state $B$ with respect to the original state of Eq. (2), obtained after an appropriate transformation performed according to the result of Alice's Bell measurement; and the bar on the right-hand side indicates averaging over the unknown coefficients $x$ and $y$. It is clear that $P_{1}=P_{2}=\frac{1}{4}, P_{5}$ $=P_{F}(x, y)$ [see Eq. (4)], $P_{3}=P_{4}=\frac{1}{4}-\frac{1}{2} P_{F}(x, y), F_{1}=F_{2}=1$, and $F_{3}=F_{4}=F_{\max }(x, y)$, where the actual maximum value $F_{\text {max }}(x, y)$ of the fidelity of Eq. (5) can be replaced by $F(x, y)$ 


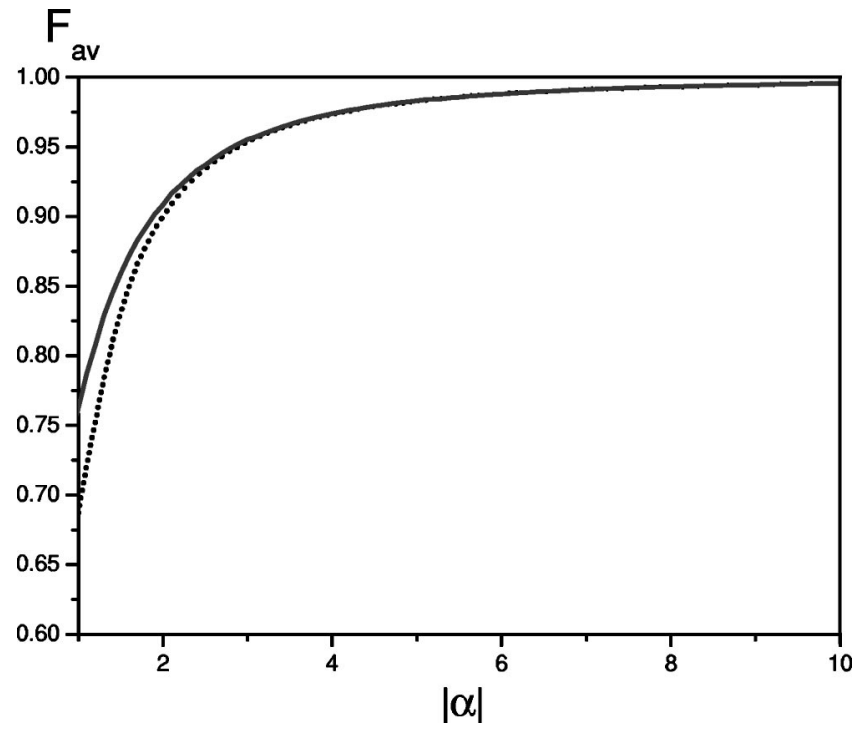

FIG. 3. The average fidelity $F_{\text {av }}$ as a function of $|\alpha|$. The solid and dotted curves represent $F_{\text {av }}$ computed using, for the value of $F_{3}=F_{4}$, the actual maximum value $F_{\text {max }}$ and the value of the fidelity at $t=\pi /|\alpha| g_{0}$, respectively.

at time $t=\pi /|\alpha| g_{0}$ if $|\alpha|$ is sufficiently large. We can also calculate easily $F_{5}$ and obtain $F_{5}=\left[\left(1-e^{-2|\alpha|^{2}}\right) / 2\right]|x-y|^{2}$. In order to perform averaging over $x$ and $y$, it is convenient to express the state $|\Psi\rangle_{c}$ of Eq. (2) in orthonormal bases. We choose to express it in terms of even and odd coherent states $\left|\alpha_{e}\right\rangle$ and $\left|\alpha_{o}\right\rangle[16]$ as

$$
|\psi\rangle_{C}=\sin \frac{\theta}{2}\left|\alpha_{e}\right\rangle_{C}+\cos \frac{\theta}{2} e^{i \phi}\left|\alpha_{o}\right\rangle_{C} .
$$

The probabilities $P_{i}$ and the fidelities $F_{i}$ can be expressed as a function of $\theta$ and $\phi$, and $P_{i}(\theta, \phi)$ and $F_{i}(\theta, \phi)$, and the averaging can then be performed according to

$$
F_{\mathrm{av}}=\int_{0}^{2 \pi} d \phi \int_{0}^{\pi} \sin \theta d \theta \sum_{i=1}^{5} P_{i}(\theta, \phi) F_{i}(\theta, \phi) .
$$

In Fig. 3, we show the average fidelity $F_{\text {av }}$, as a function of $\alpha$, that we computed using the Monte Carlo method, where the solid and dotted curves are obtained by using the actual maximum value $F_{\text {max }}$ and $F\left(t=\pi /|\alpha| g_{0}\right)$, respectively, for the value of the fidelity $F_{3}=F_{4}=F(x, y)$. Compared with the standard linear optical scheme of teleporting a superposed polarization state, where a beam splitter and two polarizing beam splitters are used for Bell-state measurements, in which case the average fidelity is given by $\frac{5}{6},{ }^{1}$ the present case yields a higher average fidelity for $|\alpha| \gtrless 1.33$. If $|\alpha|$ is not too

\footnotetext{
${ }^{1}$ When the Bell-state measurement is successful, which occurs with the probability of $\frac{1}{2}$, the fidelity is 1 . When it is not, which also occurs with the probability of $\frac{1}{2}$, the maximum possible fidelity averaged over the unknown coefficients after a bit-flip operation by Bob is $\frac{2}{3}$, being equivalent to the case when Alice and Bob are connected only by a classical channel [17]. The average fidelity is thus given by $\frac{1}{2} \times 1+\frac{1}{2} \times \frac{2}{3}=\frac{5}{6}$.
}

small, the average fidelity is close to unity (for example, $F_{\mathrm{av}}=0.955$ at $|\alpha|=3$ ), and for reasonably large $|\alpha|, F_{\mathrm{av}}$ can be regarded as practically equal to 1 .

\section{DISCUSSION}

In this section, we remark on the issue concerning experimental realization of our proposed scheme. The scheme requires generation and manipulation of the entangled coherent state, and photodetection that distinguishes between even and odd photons, all of which are highly demanding experimentally. There have, however, been suggestions and proposals that would help to overcome the difficulties. The entangled coherent state of Eq. (1) can be generated by illuminating a $50 / 50$ beam splitter with a superposed coherent state $|\sqrt{2} \alpha\rangle-|-\sqrt{2} \alpha\rangle$ through one of the input ports. The superposed coherent state can in turn be generated by exploiting nonlinear interaction between a coherent state field and atoms $[13,18]$, or by means of a conditional measurement on a beam splitter [19]. Discriminating even to odd photons requires in principle detectors with single-photon resolution that can distinguish between $n$ and $(n+1)$ photons. Visible light photon counters have recently been constructed that can distinguish between no photon and a single photon with a quantum efficiency exceeding $70 \%$ [20], and that can distinguish between a single photon and two photons with a quantum efficiency of $47 \%$ [21]. Using such counters in an arrangement of detector cascades [22] or N-ports [23], it is in principle possible to distinguish between $n$ and $(n+1)$ photons. It has also been suggested that $n$ and $(n+1)$ photons can be distinguished by utilizing homodyne detection looking at the imaginary quadrature [11], or by coupling the field to a two-level atom through nonlinear interaction [24]. Our scheme also requires the superposed coherent state to enter the cavity without destruction. This represents another experimental challenge, because the superposed coherent state is in general highly fragile.

In conclusion, we have shown that a near-complete teleportation of a superposed coherent state is possible using only a linear-optical scheme and an atom-field interaction. The average fidelity of the proposed scheme exceeds that of the standard linear-optical scheme for teleporting a superposed polarization state, as long as $|\alpha| \gtrsim 1.33$. We mention as the last remark that another advantage of the teleportation scheme using coherent state qubits is that, compared with schemes based on other types of qubits such as polarization qubits, it is in general more robust against amplitude errors that can occur during generation or transmission of entanglement [25].

\section{ACKNOWLEDGMENTS}

This research was supported by Korea Research Foundation Grant No. KRF-2002-070-C00029. We wish to thank Dr. J. Kim and Dr. B. A. Nguyen of KIAS and Professor M. S. Kim and Dr. H. Jeong of Queens University, Belfast for helpful discussions. 
[1] C. H. Bennett, G. Brassard, C. Crepeau, R. Jozsa, A. Peres, and W. K. Wootters, Phys. Rev. Lett. 70, 1895 (1993).

[2] D. Bouwmeester, J. W. Pan, K. Mattle, M. Eibl, H. Weinfurter, and A. Zeilinger, Nature (London) 390, 575 (1997); D. Boschi, S. Branca, F. De Martini, L. Hardy, and S. Popescu, Phys. Rev. Lett. 80, 1121 (1998).

[3] H. W. Lee and J. Kim, Phys. Rev. A 63, 012305 (2000); E. Lombardi, F. Sciarrino, S. Popescu, and F. De Martini, Phys. Rev. Lett. 88, 070402 (2002); S. Giacomini, F. Sciarrino, E. Lombardi, and F. De Martini, Phys. Rev. A 66, 030302(R) (2002).

[4] L. Vaidman, Phys. Rev. A 49, 1473 (1994); S. L. Braunstein and H. J. Kimble, Phys. Rev. Lett. 80, 869 (1998); A. Furusawa, J. L. Sorensen, S. L. Braunstein, C. A. Fuchs, H. J. Kimble, and E. S. Polzik, Science 282, 707 (1998).

[5] N. Lütkenhaus, J. Calsamiglia, and K. A. Suominen, Phys. Rev. A 59, 3295 (1999).

[6] E. Knill, R. Laflamme, and G. J. Milburn, Nature (London) 409, 46 (2001); P. G. Kwiat and H. Weinfurter, Phys. Rev. A 58, R2623 (1998); L. Vaidman and N. Yoran, ibid. 59, 116 (1999); M. O. Scully, B. G. Englert, and C. J. Bednar, Phys. Rev. Lett. 83, 4433 (1999); E. DelRe, B. Crosignani, and P. Di Porto, ibid. 84, 2989 (2000); D. Vitali, M. Fortunato, and P. Tombesi, ibid. 85, 445 (2000); M. G. A. Paris, M. B. Plenio, S. Bose, D. Jonathan, and G. M. D'Ariano, Phys. Lett. A 273, 153 (2000); S. P. Walborn, S. Padua, and C. H. Monken, e-print quant-ph/0307212.

[7] Y. H. Kim, S. P. Kulik, and Y. Shih, Phys. Rev. Lett. 86, 1370 (2001).

[8] X. Wang, Phys. Rev. A 64, 022302 (2001); S. J. van Enk and O. Hirota, ibid. 64, 022313 (2001); O. Hirota, S. J. van Enk, K. Nakamura, M. Shoma, and K. Kato, e-print quant-ph/ 0101096; H. Jeong, M. S. Kim, and J. Lee, Phys. Rev. A 64, 052308 (2001); Y. Zheng, Y. J. Gu, and G. C. Guo, J. Opt. B: Quantum Semiclassical Opt. 5, 29 (2003); N. B. An, Phys. Rev. A 68, 022321 (2003).

[9] B. C. Sanders, Phys. Rev. A 45, 6811 (1992).
[10] H. Jeong and M. S. Kim, e-print quant-ph/0109077.

[11] T. C. Ralph, A. Gilchrist, G. J. Milburn, W. J. Munro, and S. Glancy, e-print quant-ph/0306004.

[12] D. F. Walls and G. J. Milburn, Phys. Rev. A 31, 2403 (1985).

[13] M. Brune, E. Hagley, J. Dreyer, X. Maitre, A. Maali, C. Wunderlich, J. M. Raimond, and S. Haroche, Phys. Rev. Lett. 77, 4887 (1996).

[14] J. M. Raimond, M. Brune, and S. Haroche, Rev. Mod. Phys. 73, 565 (2001).

[15] J. Ye, D. W. Vernooy, and H. J. Kimble, Phys. Rev. Lett. 83, 4987 (1999).

[16] V. V. Dodonov, I. A. Malkin, and V. I. Man'ko, Physica (Amsterdam) 72, 597 (1974); Y. Xia and G. Guo, Phys. Lett. A 136, 281 (1989).

[17] S. Popescu, Phys. Rev. Lett. 72, 797 (1994); S. Massar and S. Popescu, ibid. 74, 1259 (1995).

[18] B. Yurke and D. Stoler, Phys. Rev. Lett. 57, 13 (1986); M. Brune, S. Haroche, J. M. Raimond, L. Davidovich, and N. Zagury, Phys. Rev. A 45, 5193 (1992).

[19] M. Dakna, T. Anhut, T. Opatrny, L. Knoll, and D. G. Welsch, Phys. Rev. A 55, 3184 (1997).

[20] P. G. Kwiat, A. M. Steinberg, R. Y. Chiao, P. H. Eberhard, and M. D. Petroff, Phys. Rev. A 48, R867 (1993); S. Takeuch, J. Kim, Y. Yamamoto, and H. H. Hogue, Appl. Phys. Lett. 74, 1063 (1999).

[21] J. Kim, S. Takeuch, Y. Yamamoto, and H. H. Hogue, Appl. Phys. Lett. 74, 902 (1999).

[22] S. Song, C. M. Caves, and B. Yurke, Phys. Rev. A 41, R5261 (1990); P. Kok and S. L. Braunstein, ibid. 63, 033812 (2001).

[23] M. Reck, A. Zeilinger, H. J. Bernstein, and P. Bertani, Phys. Rev. Lett. 73, 58 (1994).

[24] B. Yurke and D. Stoler, Phys. Rev. A 35, 4846 (1987); Phys. Rev. Lett. 79, 4941 (1997); C. C. Gerry, Phys. Rev. A 61, 043811 (2000).

[25] H. Kim, Y. W. Cheong, and H. W. Lee, Phys. Rev. A 70, 012309 (2004). 